\title{
Facial Rash following Infliximab Treatment
}

NARIN-NARD CARMEL, MD, Sackler School of Medicine, Tel Aviv University, Tel Aviv, and Department of Internal Medicine E, Meir Medical Center, Kfar Saba, Israel; YAIR LEVY, MD, Department of Internal Medicine E, Meir Medical Center. Address correspondence to Dr. Y. Levy, Department of Medicine E, Meir Medical Center, Kfar-Saba, 44261, Israel. E-mail: levy.yair@clalit.org.il. J Rheumatol 2016;43:452; doi:10.3899/jrheum.150579

In spondyloarthritis (SpA), tumor necrosis factor (TNF)- $\alpha$ antagonists are rarely linked to systemic lupus erythematosus (SLE). Usually in such cases, the biological agent is discontinued.

A 30-year-old woman with Crohn-related SpA presented with facial rash after treatment with a third dose of infliximab (IFX). She had been diagnosed with Crohn disease 3 years earlier based on a typical clinical presentation, accompanied by extraintestinal manifestations of uveitis and erythema nodosum. After 3 treatments with anti-TNF- $\alpha$, a facial rash resembling that of SLE (Figure 1, left panel) was noted. Non-deforming, symmetrical arthritis of the small joints of the hands appeared, without other organ involvement. IFX was stopped immediately.

Skin biopsy histopathology demonstrated vacuolar interface changes and perivascular inflammatory infiltrates with diffuse infiltration of lymphocytes and neutrophils. Immunofluorescence testing showed a weakly positive SLE band for IgG. Antinuclear factor shifted from negative to positive (1:160, homogeneous pattern). The patient was positive for dsDNA antibodies. Other extractable nuclear antigen antibodies were negative. The patient was treated with prednisone (20 mg once a day) with rapid tapering and hydroxychloroquine (200 bid) and azathioprine (50 mg bid), with good response.

Four months later, the rash disappeared (Figure 1, right panel), the arthritis subsided, and serologic tests were negative. Naranjo scale ${ }^{1}$ was calculated, indicating a probable adverse drug reaction. TNF- $\alpha$ antagonists are rarely associated with drug-induced SLE, a complication ${ }^{2,3}$ that usually leads to discontinuation of the biological agent.

\section{REFERENCES}

1. Naranjo CA, Busto U, Sellers EM, Sandor P, Ruiz I, Roberts EA, et al. A method for estimating the probability of adverse drug reactions. Clin Pharmacol Ther 1981;30:239-45.

2. Mounach A, Ghazi M, Nouijai A, Ghozlani I, Achemlal L, Bezza A, et al. Drug-induced lupus-like syndrome in ankylosing spondylitis treated with infliximab. Clin Exp Rheumatol 2008;26:1116-8.

3. Williams EL, Gadola S, Edwards CJ. Anti-TNF-induced lupus. Rheumatology 2009;48:716-20.
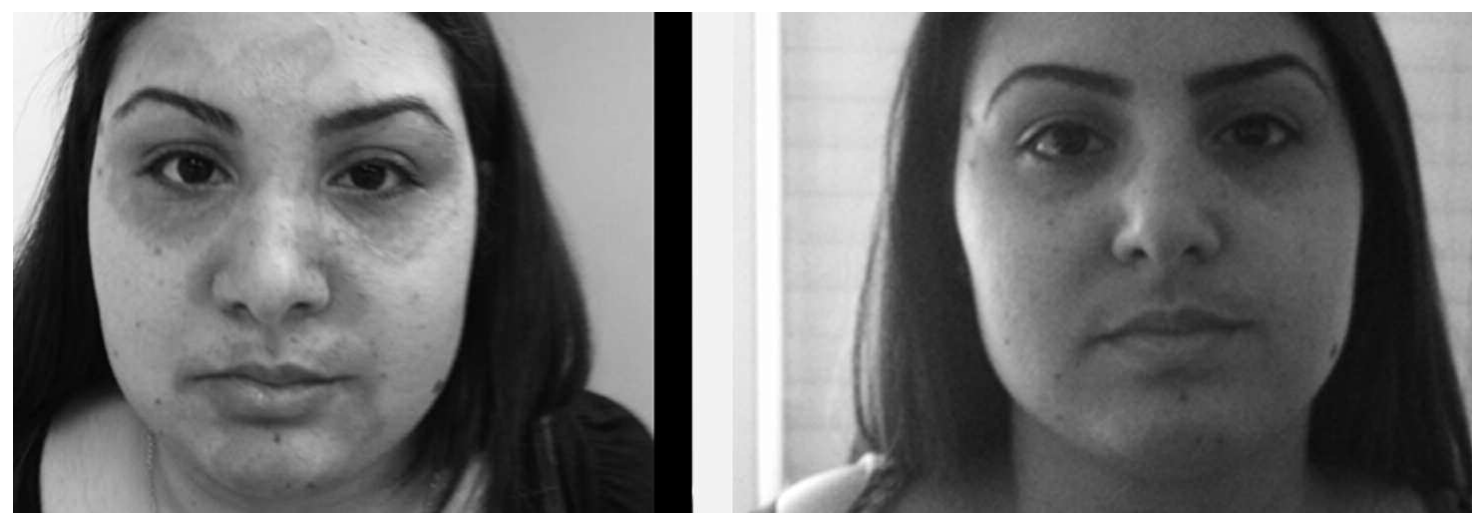

Figure 1. A patient with Crohn-related spondyloarthritis presented with a facial rash (left panel) resembling that of systemic lupus erythematosus, after 3 treatments with anti-tumor necrosis factor- $\alpha$. Four months later, after treatment with prednisone, hydroxychloroquine, and azathioprine, the rash disappeared (right panel). 